DAMTP

February 4, 2008

University of Cambridge

\title{
The Einstein Equations on the 3-Brane World
}

\author{
Tetsuya Shiromizu ${ }^{1,3,4}$, Kei-ichi Maeda ${ }^{2,5}$ and Misao Sasaki ${ }^{2,3,6}$ \\ ${ }^{1}$ DAMTP, University of Cambridge \\ Silver Street, Cambridge CB3 9EW, United Kingdom \\ ${ }^{2}$ Isaac Newton Institute, University of Cambridge, \\ 20 Clarkson Road, Cambridge CB3 OEH, United Kingdom \\ ${ }^{3}$ Department of Physics, The University of Tokyo, Tokyo 113-0033, Japan \\ ${ }^{4}$ Research Centre for the Early Universe(RESCEU), \\ The University of Tokyo, Tokyo 113-0033, Japan \\ ${ }^{5}$ Department of Physics, Waseda University, Shinjuku, Tokyo 169-8555, Japan \\ ${ }^{6}$ Department of Earth and Space Science, Graduate School of Science, \\ Osaka University, Toyonaka 560-0043, Japan
}

\begin{abstract}
We carefully investigate the gravitational equations of the brane world, in which all the matter forces except gravity are confined on the 3-brane in a 5-dimensional spacetime with $Z_{2}$ symmetry. We derive the effective gravitational equations on the brane, which reduce to the conventional Einstein equations in the low energy limit. From our general argument we conclude that the first Randall \& Sundrum-type theory (RS1) [hep-ph/9905221] predicts that the brane with the negative tension is an anti-gravity world and hence should be excluded from the physical point of view. Their second-type theory (RS2) hep-th/9906064 where the brane has the positive tension provides the correct signature of gravity. In this latter case, if the bulk spacetime is exactly anti-de Sitter, generically the matter on the brane is required to be spatially homogeneous because of the Bianchi identities. By allowing deviations from anti-de Sitter in the bulk, the situation will be relaxed and the Bianchi identities give just the relation between the Weyl tensor and the energy momentum tensor. In the present brane world scenario, the effective Einstein equations cease to be valid during an era when the cosmological constant on the brane is not well-defined, such as in the case of the matter dominated by the potential energy of the scalar field.
\end{abstract}

PACS: 04.50.+h; 98.80.Cq DAMTP-1999-150; NI99018-SFU; UTAP-349; RESCEU-40/99;

WU-AP/85/99;OUTAP-103

Recent progress in the superstring theory tells us that we are living in 11 dimensions [1], and different string theories are connected with each other via dualities. Among string theories, the 10-dimensional $E_{8} \times E_{8}$ heterotic string theory is a strong candidate for our real world because the theory may contain the standard model. Recently Horava \& Witten showed that the 10-dimensional $E_{8} \times E_{8}$ heterotic string is related to an 11-dimensional theory on the orbifold $\mathbf{R}^{10} \times \mathbf{S}^{1} / Z_{2}$ [2]. Therein the standard model particles are confined to the 4-dimensional spacetime. On the other hand, gravitons propagate in the full spacetime.

This situation can be simplified to a 5-dimensional problem where matter fields are confined to the 4-dimensional spacetime while gravity acts in 5 dimensions. In this category much work has been done. Among of all, the pioneer work in spacetime with one extra dimension has been done by Randall \& Sundrum 3. 3 . [5] where our brane is identical to a domain wall in 5-dimensional anti-de Sitter spacetime. In their first paper [3], they proposed a mechanism to solve the hierarchy problem by a small extra dimension. In their second paper [4], the brane world with a positive 
tension was investigated. Then a non-perturbative aspect of the theory was investigated [6]. The final fate of gravitational collapse was discussed in the brane world picture [7]. The inflation solution has been discovered [8] 10 . In these treatments, however, the contribution from matter excitations has not been seriously considered. Such work is partially performed in a cosmological context linked to the conventional Friedman equation [11 14. The cosmological solution associated with the heterotic string theory also has been constructed [15]. We mention work on the brane world motivated by the hierarchy problem. Before Randall \& Sundrum's work, large extra dimensions were proposed to solve the hierarchy problem [16]. The related cosmology also has been actively investigated [17].

In this paper, we derive the effective Einstein equations on the 3-brane. For simplicity the bulk spacetime is assumed to have 5 dimensions. In the beginning we do not assume any conditions on the bulk spacetime. Later, we assume the $Z_{2}$-symmetry and confinement of the matter energy momentum tensor on the brane, in accordance with the brane world scenario based on the Horava \& Witten theory [2]. The notation basically follows Wald's text [18].

In the brane world scenario, our 4-dimensional world is described by a domain wall $\left(3\right.$-brane) $\left(M, q_{\mu \nu}\right)$ in 5 dimensional spacetime $\left(V, g_{\mu \nu}\right)$. We denote the vector unit normal to $M$ by $n^{\alpha}$ and the induced metric on $M$ by $q_{\mu \nu}=g_{\mu \nu}-n_{\mu} n_{\nu}$. We start with the Gauss equation,

$$
{ }^{(4)} R_{\beta \gamma \delta}^{\alpha}={ }^{(5)} R_{\nu \rho \sigma}^{\mu} q_{\mu}^{\alpha} q_{\beta}^{\nu} q_{\gamma}{ }^{\rho} q_{\delta}^{\sigma}+K_{\gamma}^{\alpha} K_{\beta \delta}-K_{\delta}^{\alpha} K_{\beta \gamma},
$$

and the Codacci equation,

$$
D_{\nu} K_{\mu}{ }^{\nu}-D_{\mu} K={ }^{(5)} R_{\rho \sigma} n^{\sigma} q_{\mu}^{\rho}
$$

where the extrinsic curvature of $M$ is denoted by $K_{\mu \nu}=q_{\mu}{ }^{\alpha} q_{\nu}{ }^{\beta} \nabla_{\alpha} n_{\beta}, K=K_{\mu}^{\mu}$ is its trace, and $D_{\mu}$ is the covariant differentiation with respect to $q_{\mu \nu}$. Contracting the Gauss equation (1) on $\alpha$ and $\gamma$, we find

$$
{ }^{(4)} R_{\mu \nu}={ }^{(5)} R_{\rho \sigma} q_{\mu}{ }^{\rho} q_{\nu}{ }^{\sigma}-{ }^{(5)} R_{\beta \gamma \delta}^{\alpha} n_{\alpha} q_{\mu}{ }^{\beta} n^{\gamma} q_{\nu}{ }^{\delta}+K K_{\mu \nu}-K_{\mu}{ }^{\alpha} K_{\nu \alpha} .
$$

This readily gives

$$
{ }^{(4)} G_{\mu \nu}=\left[{ }^{(5)} R_{\rho \sigma}-\frac{1}{2} g_{\rho \sigma}{ }^{(5)} R\right] q_{\mu}{ }^{\rho} q_{\nu}{ }^{\sigma}+{ }^{(5)} R_{\rho \sigma} n^{\rho} n^{\sigma} q_{\mu \nu}+K K_{\mu \nu}-K_{\mu}{ }^{\rho} K_{\nu \rho}-\frac{1}{2} q_{\mu \nu}\left(K^{2}-K^{\alpha \beta} K_{\alpha \beta}\right)-\tilde{E}_{\mu \nu},
$$

where

$$
\tilde{E}_{\mu \nu} \equiv{ }^{(5)} R_{\beta \rho \sigma}^{\alpha} n_{\alpha} n^{\rho} q_{\mu}^{\beta} q_{\nu}{ }^{\sigma}
$$

Using the 5-dimensional Einstein equations,

$$
{ }^{(5)} R_{\alpha \beta}-\frac{1}{2} g_{\alpha \beta}{ }^{(5)} R=\kappa_{5}^{2} T_{\alpha \beta},
$$

where $T_{\mu \nu}$ is the 5-dimensional energy-momentum tensor, together with the decomposition of the Riemann tensor into the Weyl curvature, the Ricci tensor and the scalar curvature;

$$
{ }^{(5)} R_{\mu \alpha \nu \beta}=\frac{2}{3}\left(g_{\mu[\nu}{ }^{(5)} R_{\beta] \alpha}-g_{\alpha[\nu}{ }^{(5)} R_{\beta] \mu}\right)-\frac{1}{6} g_{\mu[\nu} g_{\beta] \alpha}{ }^{(5)} R+{ }^{(5)} C_{\mu \alpha \nu \beta},
$$

we obtain the 4-dimensional equations as

$$
{ }^{(4)} G_{\mu \nu}=\frac{2 \kappa_{5}^{2}}{3}\left(T_{\rho \sigma} q_{\mu}{ }^{\rho} q_{\nu}{ }^{\sigma}+\left(T_{\rho \sigma} n^{\rho} n^{\sigma}-\frac{1}{4} T_{\rho}^{\rho}\right) q_{\mu \nu}\right)+K K_{\mu \nu}-K_{\mu}{ }^{\sigma} K_{\nu \sigma}-\frac{1}{2} q_{\mu \nu}\left(K^{2}-K^{\alpha \beta} K_{\alpha \beta}\right)-E_{\mu \nu},
$$

where

$$
E_{\mu \nu} \equiv{ }^{(5)} C_{\beta \rho \sigma}^{\alpha} n_{\alpha} n^{\rho} q_{\mu}^{\beta} q_{\nu}{ }^{\sigma} .
$$

Note that $E_{\mu \nu}$ is traceless. From the Codacci equation (2) and the 5-dimensional Einstein equations (6), we find

$$
D_{\nu} K_{\mu}{ }^{\nu}-D_{\mu} K=\kappa_{5}^{2} T_{\rho \sigma} n^{\sigma} q_{\mu}^{\rho}
$$

So far we have not assumed any particular symmetry nor particular form of the energy momentum tensor. From now on, we take a brane world scenario. For convenience, we choose a coordinate $\chi$ such that the hypersurface $\chi=0$ coincides with the brane world and $n_{\mu} d x^{\mu}=d \chi$, which implies 


$$
a^{\mu}=n^{\nu} \nabla_{\nu} n^{\mu}=0
$$

This is a condition on the coordinate in the direction of the extra dimension. We assume this choice is possible at least in the neighbourhood of the brane, $\left(M, q_{\mu \nu}\right)$. In more explicit terms, we assume the 5-dimensional metric to have the form,

$$
d s^{2}=d \chi^{2}+q_{\mu \nu} d x^{\mu} d x^{\nu}
$$

Bearing brane world spirit in mind, we assume that the 5-dimensional energy-momentum tensor has the form

$$
T_{\mu \nu}=-\Lambda g_{\mu \nu}+S_{\mu \nu} \delta(\chi)
$$

where

$$
S_{\mu \nu}=-\lambda q_{\mu \nu}+\tau_{\mu \nu}
$$

with $\tau_{\mu \nu} n^{\nu}=0 . \Lambda$ is the cosmological constant of the bulk spacetime. $\lambda$ and $\tau_{\mu \nu}$ are the vacuum energy and the energy-momentum tensor, respectively, in the brane world. Note that $\lambda$ is the tension of the brane in 5 dimensions. Properly speaking $S_{\mu \nu}$ should be evaluated by the variational principle of the 4-dimensional Lagrangian for matter fields because the normal matter except for gravity is assumed to be living only in the $\chi=0$ brane. It should be noted that the decomposition of $S_{\mu \nu}$ into $\lambda q_{\mu \nu}$ and $\tau_{\mu \nu}$ can be ambiguous, particularly in cosmological contexts.

The singular behaviour in the energy-momentum tensor leads us to the so-called Israel's junction condition [19],

$$
\begin{aligned}
{\left[q_{\mu \nu}\right] } & =0 \\
{\left[K_{\mu \nu}\right] } & =-\kappa_{5}^{2}\left(S_{\mu \nu}-\frac{1}{3} q_{\mu \nu} S\right),
\end{aligned}
$$

where $[X]:=\lim _{\chi \rightarrow+0} X-\lim _{\chi \rightarrow-0} X=X^{+}-X^{-}$.

Now we impose the $Z_{2}$-symmetry on this spacetime, with the brane as the fixed point. Interestingly the symmetry uniquely determines the extrinsic curvature of the brane in terms of the energy momentum tensor,

$$
K_{\mu \nu}^{+}=-K_{\mu \nu}^{-}=-\frac{1}{2} \kappa_{5}^{2}\left(S_{\mu \nu}-\frac{1}{3} q_{\mu \nu} S\right) .
$$

Hereafter we focus our attention on quantities evaluated on the brane. Because of the $Z_{2}$-symmetry, we may evaluate quantities either on the + or - side of the brane. Hence we omit the indices \pm below for brevity.

Substituting Eq. (16) into Eq. (8), we obtain the gravitational equations on the 3-brane in the form,

$$
{ }^{(4)} G_{\mu \nu}=-\Lambda_{4} q_{\mu \nu}+8 \pi G_{N} \tau_{\mu \nu}+\kappa_{5}^{4} \pi_{\mu \nu}-E_{\mu \nu}
$$

where

$$
\begin{aligned}
\Lambda_{4} & =\frac{1}{2} \kappa_{5}^{2}\left(\Lambda+\frac{1}{6} \kappa_{5}^{2} \lambda^{2}\right), \\
G_{N} & =\frac{\kappa_{5}^{4} \lambda}{48 \pi} \\
\pi_{\mu \nu} & =-\frac{1}{4} \tau_{\mu \alpha} \tau_{\nu}{ }^{\alpha}+\frac{1}{12} \tau \tau_{\mu \nu}+\frac{1}{8} q_{\mu \nu} \tau_{\alpha \beta} \tau^{\alpha \beta}-\frac{1}{24} q_{\mu \nu} \tau^{2},
\end{aligned}
$$

and $E_{\mu \nu}$ is the part of the 5-dimensional Weyl tensor defined in Eq. (9). It should be noted that $E_{\mu \nu}$ in the above is the limiting value at $\chi=+0$ or -0 but not the value exactly on the brane. This is our main result. It resembles the conventional Einstein equations in 4 dimensions. In fact, the Einstein equations can be recovered by taking the limit $\kappa_{5} \rightarrow 0$ while keeping $G_{N}$ finite. Nevertheless there are some important differences. As can be easily seen, the existence of Newton's gravitational constant $G_{N}$ strongly relies on the presence of the vacuum energy $\lambda$. In other words, it becomes impossible to define Newton's gravitational constant during an era when the distinction between the vacuum energy and the normal matter energy is ambiguous. Furthermore, we would have the wrong sign of $G_{N}$ if $\lambda<0$ [12]. The $\pi_{\mu \nu}$ term, which is quadratic in $\tau_{\mu \nu}$ could play a very important role, especially in the early universe when the matter energy scale is high 11,13 .

In addition to these features that have been pointed out previously, Eq. (17) contains a new term, $E_{\mu \nu}$. It is a part of the 5-dimensional Weyl tensor and carries information of the gravitational field outside the brane. It is non-vanishing 
if the bulk spacetime is not purely anti-de Sitter. At the same time, it is not freely specifiable but is constrained by the motion of the matter on the brane. Let us show this feature now. Together with Eq. (16), Eq. (10) implies the conservation law for the matter,

$$
D_{\nu} K_{\mu}^{\nu}-D_{\mu} K \propto D_{\nu} \tau_{\mu}^{\nu}=0
$$

Therefore the contracted Bianchi identities $D^{\mu(4)} G_{\mu \nu}=0$ imply the relation between $E_{\mu \nu}$ and $\tau_{\mu \nu}$ as

$$
\begin{aligned}
D^{\mu} E_{\mu \nu} & =K^{\alpha \beta}\left(D_{\nu} K_{\alpha \beta}-D_{\beta} K_{\nu \alpha}\right) \\
& =\frac{1}{4} \kappa_{5}^{4}\left[\tau^{\alpha \beta}\left(D_{\nu} \tau_{\alpha \beta}-D_{\beta} \tau_{\nu \alpha}\right)+\frac{1}{3}\left(\tau_{\mu \nu}-q_{\mu \nu} \tau\right) D^{\mu} \tau\right] .
\end{aligned}
$$

Thus $E_{\mu \nu}$ is not freely specifiable but its divergence is constrained by the matter term. If one further decomposes $E_{\mu \nu}$ into the transverse-traceless part, $E_{\mu \nu}^{T T}$, and the longitudinal part, $E_{\mu \nu}^{L}$, the latter is determined completely by the matter. Hence if the $E_{\mu \nu}^{T T}$ part is absent, the equations will be closed solely with quantities that reside in the brane. However, as usually the case in the conventional gravity, the $E_{\mu \nu}^{T T}$ part corresponds to gravitational waves or gravitons in 5 dimensions, and they will be inevitably excited by matter motions and their excitations affect matter motions in return. This implies the effective gravitational equations on the brane are not closed but one must solve the gravitational field in the bulk at the same time in general. Since the derivation of equations that govern the evolution of $E_{\mu \nu}^{T T}$ is technically complicated, we defer it to Appendix A.

Let us now estimate the effect of each term on the right-hand side of Eq. (17). We set $\kappa_{5}^{-2}=M_{G}^{3}$ and $\lambda=M_{\lambda}^{4}$, and assume $\Lambda=O\left(\kappa_{5}^{2} \lambda^{2}\right)$. It should be noted that these do not have to be planck scale quantities. One can scale them as $M_{G} \rightarrow f^{2} M_{G}$ and $M_{\lambda} \rightarrow f^{3} M_{\lambda}$, where $f$ is an arbitrary constant, while keeping the gravitational constant $G_{N}$ unchaged. Nevertheless, here we assume $M_{G}$ and $M_{\lambda}$ to be sufficiently large compared to the characteristic energy scale of the matter which we denote by $M$.

The first term on the right-hand side of Eq. (17) is the net cosmological constant in 4 dimensions. It is assumed that $\Lambda<0$. Hence $\Lambda_{4}$ may take arbitrary value as one may wish by appropriately specifying the values of $\Lambda$ and $\lambda$. The second term is the contribution from normal matters which should satisfy the local energy condition (assuming the decomposition of $S_{\mu \nu}$ into $\lambda$ and $\tau_{\mu \nu}$ is well-defined). The $\pi_{\mu \nu}$ term which is quadratic in $\tau_{\mu \nu}$ is expected to be negligible in the low energy limit. In fact, the ratio of these terms to the third term is approximately given by

$$
\frac{\kappa_{5}^{4}\left|\pi_{\mu \nu}\right|}{G_{N}\left|\tau_{\mu \nu}\right|} \sim \frac{\kappa_{5}^{4}\left|\tau_{\mu \alpha} \tau_{\nu}{ }^{\alpha}+\cdots\right|}{G_{N}\left|\tau_{\mu \nu}\right|} \sim \frac{M^{4}}{M_{\lambda}^{4}}
$$

We now turn to the Weyl tensor part. First let us consider the longitudinal part $E_{\mu \nu}^{L}$. Since it is determined by $\tau_{\mu \nu}$ through Eq. (22), we have

$$
\frac{\left|E_{\mu \nu}^{L}\right|}{G_{N}\left|\tau_{\mu \nu}\right|} \sim \frac{\kappa_{5}^{4}\left|\tau_{\mu \alpha} \tau_{\nu}{ }^{\alpha}+\cdots\right|}{G_{N}\left|\tau_{\mu \nu}\right|} \sim \frac{M^{4}}{M_{\lambda}^{4}}
$$

This is the same order of magnitude as the $\pi_{\mu \nu}$ term. Second, we consider the $E_{\mu \nu}^{T T}$ part. We focus on the effect due to matter excitations on the brane. Here we borrow the discussion of [4] to evaluate the leading order of magnitude of its effect. The gravitational potential between two bodies on the brane is modified via exchange of gravitons living in 5 dimensions as 4

$$
V(r) \sim \frac{G_{N} m_{1} m_{2}}{r}\left(1+\frac{1}{r^{2} k^{2}}\right)
$$

where $r$ is the distance between the two bodies and $k=\kappa_{5}^{2} \lambda / 6$. Since this effect must be contained in Eq. (17), it should appear in the $E_{\mu \nu}$ term. Therefore as a conservative estimate, we obtain

$$
\frac{\left|E_{\mu \nu}\right|}{G_{N}\left|\tau_{\mu \nu}\right|} \sim \frac{M^{2}}{k^{2}} \sim \frac{M_{G}^{6} M^{2}}{M_{\lambda}^{8}}
$$

Thus $E_{\mu \nu}$ is also negligible in the low energy world. It is, however, worth noting that this term is larger than the terms quadratic in $\tau_{\mu \nu}$. The deviation from the ordinary Einstein equations in 4-dimensions first appears from gravitational excitations in the bulk spacetime. From the above estimations we conclude that the effective gravitational 
equation (17) on the brane reduce to the 4-dimensional conventional Einstein gravity, ${ }^{(4)} G_{\mu \nu} \simeq-\Lambda_{4} q_{\mu \nu}+8 \pi G_{N} \tau_{\mu \nu}$, in the low energy limit. The presence of a well-defined cosmological constant $\lambda$ is obviously essential here. 7

Finally, we note an outcome of the constraint (22). We consider the case when the bulk spacetime is pure anti-de Sitter with $E_{\mu \nu}=0$ and investigate the condition on the matter on the brane. For simplicity, we assume the perfect fluid form for the energy momentum tensor:

$$
\tau^{\mu \nu}=\rho t^{\mu} t^{\nu}+P h^{\mu \nu}
$$

where $h^{\mu \nu}=q^{\mu \nu}+t^{\mu} t^{\nu}$. The quadratic term $\pi^{\mu \nu}$ in the 4-dimensional effective gravitational equations (17) then becomes

$$
\pi^{\mu \nu}=\frac{1}{12} \rho\left(\rho t^{\mu} t^{\nu}+(\rho+2 P) h^{\mu \nu}\right)
$$

The normal conservation law $D_{\nu} \tau^{\mu \nu}=0$ implies

$$
t^{\mu} D_{\mu} \rho+(\rho+P) D_{\mu} t^{\mu}=0 \quad \text { and } \quad(\rho+P) t^{\nu} D_{\nu} t^{\mu}+h^{\mu \nu} D_{\nu} P=0 .
$$

If $E_{\mu \nu}=0$, the 4-dimensional Bianchi identities imply $D_{\nu} \pi^{\mu \nu}=0$, which gives

$$
D_{\nu} \pi^{\mu \nu}=\frac{1}{6}(\rho+P) h^{\mu \nu} D_{\nu} \rho=0 .
$$

This means $\partial_{i} \rho=0$. Hence an inhomogeneous perfect fluid is rejected.

We briefly summarize the present work. We first derived the effective 4-dimensional gravitational equations in 5 dimensions, Eq. (8), without any particular assumptions specific to the brane world scenario. Then based on the brane world scenario, we introduced the $Z_{2}$ symmetry and assumed that the matter lives only on the brane, and derived the 4-dimensional effective gravitational equations on the brane, Eq. (17). The equation tells us that a normal gravitational theory can be obtained on the brane only if the tension is positive, while an RS1-type theory [3] in which the brane has negative tension is rejected from the physical point of view (see also [12] for Friedmann cases). In the case of the brane with positive tension, the Einstein gravity is recovered in the low energy limit. Placing the brane in the 5-dimensional exact anti-de Sitter spacetime imposes a strong condition on the matter in 4-dimensions. In particular, if the matter energy-momentum tensor has the perfect fluid form, only spatially homogeneous universes are allowed. Conversely, this means that the deviation of the bulk spacetime from the exact anti-de Sitter spacetime is essential to describe our real world with matter fields.

\section{ACKNOWLEDGEMENTS}

TS would like to thank Gary W. Gibbons and DAMTP relativity group for their hospitality at Cambridge. Substantial part of this work was done while KM and MS were participating the program, "Structure Formation in the Universe", at the Newton Institute, University of Cambridge. We are grateful to the Newton Institute for their hospitality. TS's work is supported by JSPS Postdoctal Fellowship for Research Abroad. KM's work is supported in part by Monbusho Grant-in Aid for Specially Promoted Research No. 08102010. MS's work is supported in part by Monbusho Grant-in-Aid for Scientific Research No. 09640355.

\section{APPENDIX A:}

We derive the evolution equation of $E_{\mu \nu}$ to make our system of equations closed. First, we write down the Weyl tensor formulas. The $n$-dimensional Riemann tensor is written in terms of the Weyl and Ricci tensors as

$$
{ }^{(n)} R_{\alpha \beta \mu \nu}={ }^{(n)} C_{\alpha \beta \mu \nu}+\frac{2}{n-2}\left({ }^{(n)} R_{\alpha[\mu} g_{\nu] \beta}-{ }^{(n)} R_{\beta[\mu} g_{\nu] \alpha}\right)-\frac{2}{(n-1)(n-2)}^{(n)} R g_{\alpha[\mu} g_{\nu] \beta} .
$$

\footnotetext{
${ }^{*}$ As we can see from the first term in Eq. (8), the reduction to the normal Einstein gravity is also possible with the introduction of non-trivial bulk energy-momentum tensor [20].
} 
We decompose the Weyl tensor into the 'electric' and 'magnetic' parts:

$$
E_{\mu \nu} \equiv{ }^{(n)} C_{\mu \alpha \nu \beta} n^{\alpha} n^{\beta},
$$

and

$$
B_{\mu \nu \alpha} \equiv q_{\mu}^{\rho} q_{\nu}^{\sigma(n)} C_{\rho \sigma \alpha \beta} n^{\beta}
$$

$B_{\mu \nu \alpha}$ and $E_{\mu \nu}$ have the symmetry,

$$
\begin{aligned}
& B_{\alpha \beta \mu}=-B_{\beta \alpha \mu}, \quad B_{[\alpha \beta \mu]}=0, \quad B^{\alpha}{ }_{\beta \alpha}=0 . \\
& E_{\alpha \beta}=E_{\beta \alpha}, \quad E^{\alpha}{ }_{\alpha}=0 .
\end{aligned}
$$

The algebraic degrees of freedom are

$$
\begin{aligned}
& { }^{(n)} R_{\alpha \beta \mu \nu} \cdots \frac{n^{2}\left(n^{2}-1\right)}{12}, \quad{ }^{(n)} C_{\alpha \beta \mu \nu} \cdots \frac{(n-3) n(n+1)(n+2)}{12}, \\
& { }^{(n)} R_{\mu \nu} \cdots \frac{n(n+1)}{2}, \quad{ }^{(n-1)} C_{\alpha \beta \mu \nu} \cdots \frac{(n-4)(n-1) n(n+1)}{12}, \\
& B_{\alpha \beta \mu} \cdots \frac{(n-3)(n-1)(n+1)}{3}, \quad E_{\alpha \beta} \cdots \frac{(n-2)(n+1)}{2} .
\end{aligned}
$$

The $n$-dimensional Weyl tensor can be written in terms of ${ }^{(n-1)} C_{\alpha \beta \mu \nu}, E_{\alpha \beta}, B_{\mu \nu \alpha}$ and the extrinsic curvature $K_{\mu \nu}$,

$$
\begin{aligned}
{ }^{(n)} C_{\alpha \beta \mu \nu}= & { }^{(n-1)} C_{\alpha \beta \mu \nu}+2 B_{\alpha \beta[\mu} n_{\nu]}+2 B_{\mu \nu[\alpha} n_{\beta]} \\
& +\left(2 E_{\alpha[\mu} n_{\nu]} n_{\beta}-2 E_{\beta[\mu} n_{\nu]} n_{\alpha}\right)-\frac{1}{n-3}\left(2 E_{\alpha[\mu} q_{\nu] \beta}-2 E_{\beta[\mu} q_{\nu] \alpha}\right) \\
& -f_{\alpha \beta \mu \nu}+\frac{2}{n-3}\left(q_{\alpha[\mu} f_{\nu] \beta}-q_{\beta[\mu} f_{\nu] \alpha}\right)-\frac{2}{(n-2)(n-3)} f_{\sigma}^{\sigma} q_{\alpha[\mu} q_{\nu] \beta},
\end{aligned}
$$

where $q_{\mu \nu}=g_{\mu \nu}-n_{\mu} n_{\nu}$ and

$$
\begin{aligned}
f_{\alpha \beta \mu \nu} & \equiv K_{\alpha \mu} K_{\beta \nu}-K_{\alpha \nu} K_{\beta \mu}, \\
f_{\mu \nu} & \equiv f_{\mu}{ }^{\sigma}{ }_{\nu \sigma}=f^{\sigma}{ }_{\mu \sigma \nu}=K K_{\mu \nu}-K_{\mu \sigma} K^{\sigma}{ }_{\nu}=f_{\nu \mu}, \\
f^{\mu}{ }_{\mu} & =f^{\mu \nu}{ }_{\mu \nu}=K^{2}-K^{\mu \nu} K_{\mu \nu} .
\end{aligned}
$$

From now on we set $n=5$ and derive the evolution equations of $E_{\mu \nu}$ from the 5-dimensional Bianchi identities. We assume $a^{\mu}=n^{\alpha} \nabla_{\alpha} n^{\mu}=0$. For convenience, we define $\tilde{E}_{\mu \nu}$ and $\tilde{B}_{\mu \nu \alpha}$ from the Riemann tensor,

$$
\begin{aligned}
\tilde{E}_{\mu \nu} & \equiv{ }^{(5)} R_{\mu \alpha \nu \beta} n^{\alpha} n^{\beta}=-£_{n} K_{\mu \nu}+K_{\mu \alpha} K_{\nu}^{\alpha}, \\
\tilde{B}_{\mu \nu \alpha} & \equiv q_{\mu}^{\beta} q_{\nu}^{\sigma(5)} R_{\beta \sigma \alpha \rho} n^{\rho}=2 D_{[\mu} K_{\nu] \alpha} .
\end{aligned}
$$

These are related to $E_{\mu \nu}$ and $B_{\mu \nu \alpha}$ as

$$
\begin{aligned}
E_{\mu \nu} & =\tilde{E}_{\mu \nu}-\frac{1}{3} q_{\mu \nu}{ }^{(5)} R_{\alpha \beta} n^{\alpha} n^{\beta}-\frac{1}{3} q_{\mu}^{\alpha} q_{\nu}^{\beta(5)} R_{\alpha \beta}+\frac{1}{12} q_{\mu \nu}{ }^{(5)} R \\
& =-\frac{1}{3}\left({ }^{(4)} R_{\mu \nu}-\frac{1}{4} q_{\mu \nu}{ }^{(4)} R\right)-\frac{2}{3} £_{n}\left(K_{\mu \nu}-\frac{1}{4} q_{\mu \nu} K\right)+\frac{1}{3} K_{\mu \alpha} K_{\nu}^{\alpha}+\frac{1}{4} q_{\mu \nu}\left(K_{\alpha \beta} K^{\alpha \beta}-\frac{1}{3} K^{2}\right), \\
B_{\mu \nu \alpha} & =\tilde{B}_{\mu \nu \alpha}+\frac{2}{3}\left(D_{\beta} K_{[\mu}^{\beta}-D_{[\mu} K\right) q_{\nu]}^{\alpha} \\
& =2 D_{[\mu} K_{\nu] \alpha}+\frac{2}{3}\left(D_{\beta} K_{[\mu}^{\beta}-D_{[\mu} K\right) q_{\nu]}^{\alpha} .
\end{aligned}
$$

The 5-dimensional Bianchi identities are

$$
\nabla_{[\mu}^{(5)} R_{\nu \alpha] \beta \sigma}=0,
$$

from which we obtain the following four sets of identities: 


$$
\begin{gathered}
D_{[\mu} \tilde{B}_{\nu \alpha]}{ }^{\beta}+K_{[\mu}^{\sigma(4)} R_{\nu \alpha] \sigma}{ }^{\beta}=0, \\
£_{n} \tilde{B}_{\mu \nu \alpha}+2 D_{[\mu} \tilde{E}_{\nu] \alpha}-K_{\alpha}^{\sigma} \tilde{B}_{\mu \nu \sigma}+2 \tilde{B}_{\alpha \sigma[\mu} K_{\nu]}^{\sigma}=0, \\
£_{n}^{(4)} R_{\mu \nu \alpha \beta}+2^{(4)} R_{\mu \nu \sigma[\alpha} K_{\beta]}^{\sigma}+2 D_{[\mu} \tilde{B}_{|\alpha \beta| \nu]}=0, \\
D_{[\mu}{ }^{(4)} R_{\nu \alpha] \beta \sigma}=0 .
\end{gathered}
$$

From Eq. A11 and the Israel's junction condition, we obtain

$$
\left[\tilde{B}_{\mu \nu \alpha}\right]=2 D_{[\mu}\left[K_{\nu] \alpha}\right]=-2 \kappa_{5}^{2} D_{[\mu}\left(\tau_{\nu] \alpha}-\frac{1}{3} q_{\nu] \alpha} \tau\right) .
$$

Thus the $Z_{2}$-symmetry uniquely determines the value of $B_{\mu \nu \alpha}$ on the brane as

$$
\begin{aligned}
\tilde{B}_{\mu \nu \alpha}^{+} & =-\tilde{B}_{\mu \nu \alpha}^{-} \\
& =-\kappa_{5}^{2} D_{[\mu}\left(\tau_{\nu] \alpha}-\frac{1}{3} q_{\nu] \alpha} \tau\right), \\
B_{\mu \nu \alpha}^{+} & =2 D_{[\mu} K_{\nu] \alpha}^{+}+\frac{2}{3}\left(D_{\beta} K_{[\mu}^{+\beta}-D_{[\mu} K^{+}\right) q_{\nu] \alpha} \\
& =\tilde{B}_{\mu \nu \alpha}^{+} .
\end{aligned}
$$

These equations give the boundary conditions on the brane when one solves the evolution of $E_{\mu \nu}$ in 5 dimensions.

The equations that govern the evolution of $E_{\mu \nu}$ in the bulk (i.e., in the spacetime region away from the brane) are obtained as follows. Using the 5-dimensional Einstein equations (6), Eq. (A14) yields

$$
£_{n} B_{\mu \nu \alpha}=-2 D_{[\mu} E_{\nu] \alpha}+K_{\alpha}^{\sigma} B_{\mu \nu \sigma}-2 B_{\alpha \sigma[\mu} K_{\nu]}^{\sigma},
$$

in the bulk. Also, using Eq. (6) and (8), Eq. (A15) gives

$$
\begin{aligned}
£_{n} E_{\alpha \beta}= & D^{\mu} B_{\mu(\alpha \beta)}+\frac{1}{6} \kappa_{5}^{2} \Lambda\left(K_{\alpha \beta}-q_{\alpha \beta} K\right)+K^{\mu \nu(4)} R_{\mu \alpha \nu \beta} \\
& +3 K_{(\alpha}^{\mu} E_{\beta) \mu}-K E_{\alpha \beta}+\left(K_{\alpha \mu} K_{\beta \nu}-K_{\alpha \beta} K_{\mu \nu}\right) K^{\mu \nu},
\end{aligned}
$$

in the bulk. Together with the 4-dimensional Einstein equations (8) in the bulk, Eqs. (A19) and (A20) form a closed system of equations. In particular, one may easily recognize the wave-like character of the transverse part of $E_{\mu \nu}$, which propagates as gravitons in 5 dimensions.

[1] J. Polchinski, String Theory I \& II (Cambridge Univ. Press, Cambridge, 1998).

[2] P. Horava and E. Witten, Nucl. Phys. B460, 506 (1996); ibid B475, 94 (1996)

[3] L. Randall and R. Sundrum, hep-ph/9905221.

[4] L. Randall and R. Sundrum, hep-th/9906064.

[5] For earlier work on this topic, see

V. A. Rubakov and M. E. Shaposhnikov, Phys. Lett. 152B,136 (1983);

M. Visser, Phys. Lett. B159,22(1985);

K. Akama, Prog. Theor. Phys. 78,184(1987);

M. Gogberashvili, Mod. Phys. Lett. A14, 2025(1999); hep-ph/9908347.

[6] A. Chamblin and G. W. Gibbons, hep-th/9909130.

[7] A. Chamblin, S. W. Hawking and H. S. Reall, hep-th/9909205.

[8] N. Kaloper, hep-th/9905210.

[9] T. Nihei, hep-ph/9905487. 
[10] H. B. Kim and H. D. Kim, hep-th/9909053

[11] P. Binetruy, C. Deffayet and D. Langlois, hep-th/9905012.

[12] C. Csaki, M. Graesser, C. Kold and J. Terning, hep-ph/9906513.

[13] J. M. Cline, C. Grojean and G. Servant, hep-ph/9906523.

[14] M. Cvetic and H. Soleng, Phys. Rept. 282159(1997); K. Behrndt and M. Cvetic, hep-th/9909058.

[15] K. Benakli, Int. J. Mod. Phys. D8, 153 (1999); Phys. Lett. B447, 51 (1999);

A. Lukas, B. A. Ovrut, K.S. Stelle and D. Waldram, Phys. Rev. D59, 086001 (1999);

H. S. Reall, Phys. Rev. D59, 103506 (1999);

A. Lukas, B. A. Ovrut and D. Waldram, Phys. Rev. D60, 086001 (1999); hep-th/9902071;

H. A. Chamblin and H. S. Reall, hep-th/9903225

[16] N. Arkani-Hamed, S. Dimopoulos and G. Dvali, Phys. Lett. B424, 263 (1998);

I. Antoniadis, N. Arkani-Hamed, S. Dimopoulos and G. Dvali, Phys. Lett. B436, 257 (1998).

[17] N. Arkani-Hamed, S. Dimopoulos and G. Dvali, Phys. Rev. D59, 086004 (1999);

N. Arkani-Hamed, S. Dimopoulos, N. Kaloper, J. March-Russell, hep-ph/9903224.

[18] R. M. Wald, General Relativity, (Univ. Chicago Press, Chicago, 1984).

[19] W. Israel, Nuovo Cim. 44B, 1 (1966).

[20] P. Kanti, I. I. Kogan, K. A. Olive and M. Prospelov, hep-ph/9909481. 